\title{
Multi-objective Resource Allocation for mmWave MEC Offloading under Competition of Communication and Computing Tasks
}

\author{
Zhongling Zhao, Jia Shi, Member, IEEE, Zan Li, Senior Member, IEEE, Jiangbo Si, Member, IEEE, \\ Pei Xiao, Senior Member, IEEE, and Rahim Tafazolli, Senior Member, IEEE
}

\begin{abstract}
Towards 6G networks, such as virtual reality (VR) applications, Industry 4.0 and automated driving, demand mobile edge computing (MEC) techniques to offload computing tasks to nearby servers, which however causes fierce competition with traditional communication services. On the other hand, by introducing millimeter wave (mmWave) communication, it can significantly improve the offloading capability of MEC, so that enabling low latency and high throughput. For this sake, this paper investigates the resource management for the offload transmission of mmWave MEC system, when considering the data transmission demands from both communication-oriented users (CM-UEs) and computingoriented users (CP-UEs). In particular, the joint consideration of user pairing, beamwidth allocation and power allocation is formulated as a multi-objective problem (MOP), which includes minimizing the offloading delay of CP-UEs and maximizing the transmission rate of CM-UEs. By using $\epsilon$-constraint approach, the MOP is converted into a single-objective optimization problem (SOP) without losing Pareto optimality, and then the three-stage iterative resource allocation algorithm is proposed. Our simulation results show that, the gap between Pareto front generated by three-stage iterative resource allocation algorithm and the real Pareto front less than $0.16 \%$. Futher, the proposed algorithm with much lower complexity can achieve the performance similar to the benchmark scheme of NSGA-II, while significantly outperforms the other traditional schemes.
\end{abstract}

Index Terms-MmWave, MEC offloading, multi-objective optimization.

\section{INTRODUCTION}

In the $\mathrm{B} 5 \mathrm{G}$ and $6 \mathrm{G}$ era, the large-scale access of mobile terminals brings huge challenges to delay-sensitive demand of communication [1]. As the volume of user (UE) computing tasks rapidly increases, mobile edge computing (MEC) was developed to distribute the computing resources at the network edge in the vicinity of end-UEs in order to effectively reduce time delay and support large-scale UE access $[2,3]$.

The current research on MEC technique mainly includes network architecture, offloading decision-making, and mo-

This work is supported by the National Natural Science Foundation of China (NSFC) (No. 61901327, No. 61825104, No. 61971337 and No.62001359), the Fundamental Research Funds for the Central Universities (No. JB210109) and the Foundation of State Key Laboratory of Integrated Services Networks of Xidian University under Grant ISN22-03.

Z. Zhao, J. Shi, Z, Li, and J. Si are with the State Key Laboratory of Integrated Service Networks, School of Telecommunications Engineering, Xidian University, Xi'an, 710071, China. (email:_zhonglingzhao@stu.xidian.edu.cn;_ jiashi@xidian.edu.cn; zanli@xidian.edu.cn; jbsi@xidian.edu.cn.)

P. Xiao, and R. Tafazolli are with the Institute for Communication Systems (ICS), University of Surrey, Guildford GU2 7XH, U.K. (e-mail p.xiao@surrey.ac.uk; r.tafazolli@ surrey.ac.uk).

Copyright (c) 2021 IEEE. Personal use of this material is permitted. However, permission to use this material for any other purposes must be obtained from the IEEE by sending a request to pubs-permissions@ieee.org. bility management. Specifically, the limitation of communication resources and computing resources contradicts the explosive growth of mobile terminals, which stimulates the study of resource allocation for MEC offloading. At present, many scholars have carried out detailed research on resource allocation in traditional ultrahigh frequency (UHF) MEC environments [4-11]. The authors in [4] studied the joint computation offloading and resource allocation strategy in multi-user and multi-server scenarios. In order to overcome the problem of small continuous bandwidth available for $\mathrm{UH}_{-}$ F, some scholars combined non-orthogonal multiple access (NOMA) and MEC technology, such as [5] and [6]. The studies of [7-9] have combined MEC with other popular scenarios such as vehicular networks and unmanned aerial vehicle (UAV) networks. In addition, S. Yu et al. proposed a framework for multi-user computing results sharing in [10] and [11], and effectively reduced the offloading delay and energy consumption of MEC system. However, the limited available spectrum resources of UHF pose a strict-limit on the volume of UEs access and energy efficiency.

As a promising technology, mmWave has recently attracted large research interest because of the huge resources of available bandwidth, which can exponentially increase the transmission ability of MEC offloading [12-15], which is now become an important research trend for the $\mathrm{B} 5 \mathrm{G}$ and $6 \mathrm{G}$ era.

\section{A. Related Works}

More recently, the current studies have proposed a vision for the mmWave MEC system and conducted related research [16-20]. In [16], authors introduced mmWave MEC technology to panoramic virtual reality video (PVRV) streaming system. G. K. Tran et al. proposed an architecture of mmWave MEC system in [17], which focused on 5G Phase 2 features and ultra high speed low latency communications (uHSLLC) applications. In [18], they combined mmWave MEC and vehicular networks. Further, S. Barbarossa et al. showed how to take advantage of preliminary estimation of blocking probabilities and proposed two strategies to overcome the effect of blocking $[19,20]$. In general, the above literature that some scholars have built the framework of the mmWave MEC system, and have proved the feasibility of combining mmWave with MEC.

So far, there are very limited studies [21-24] on the resource management for mmWave MEC offloading. In order to minimize the offloading delay $[21,22]$, C. Zhao et al. conducted resource management research for mmWave MEC system, they proposed the algorithm based on the penalty dual decomposition (PDD) technique and generalized Benders 
decomposition (GBD) algorithm to solve the beamforming and UAV trajectory design respectively. In [23], authors considered multi-UE uplink scenario, in which all UEs had the same requirement: offloading of computing tasks, which aimed to minimize the total energy consumption of all UEs and obtained the suboptimal solution with lower complexity. Finally, in [24], N. Nouri et al. considered the trade-off between offloading delay and energy consumption in ultra dense mmWave NOMA MEC system. Unfortunately, the current works only considered the mmWave MEC scenario of existing offloading tasks while ignoring other traditional communication services.

\section{B. Motivation and Contribution}

In practical mmWave MEC systems, computing-oriented users (CP-UEs) and communication-oriented UEs (CM-UEs) usually coexist, and they often demand data transmission at the same time. The CP-UEs usually refer to the users who have computing tasks which need to be offloaded to MEC servers. The communication of CP-UE is generated by computing needs, which has strong timeliness. By contrast, the CM-UEs usually refer to the users who mainly care about data rate. For instance, in $5 \mathrm{G}$ or future $6 \mathrm{G}$ networks, the users demanding high-volume of computing services, such as self-driving cars and VR/AR equipments, can be seen as CP-UEs, while the users demanding high communication qualities, such as eMBB services, can be refered to CMUEs. In this case, CP-UEs and CM-UEs will compete for the same communication resources to accomplish their own tasks, which further complicates the problem of resource allocation for MEC offloading. In this paper, we invesigate the resource allocation for the mmWave MEC offloading system.

The main contribution of this paper are summarized as follows.

1) We build a novel MEC offloading framework with the capability of mmWave communication, where both the CPUEs and CM-UEs are involved. Further, we formulate the MOP by jointly considering CP-UE assignment, beamwidth selection, power allocation.

2) We propose a low complexity three-stage iterative algorithm, by means of $\epsilon$-constraint method, where the MOP is converted to the single-objective problem (SOP). Specifically, the block coordinate descent (BCD) based beamwidth and power assignment and matching theory based UE and time slot (TS) pairing are developed.

3) The intelligent algorithm, namely NSGA-II, is designed as a benchmark scheme. Further, we analyze the complexity of the proposed algorithm and the NSGA-II algorithm, and show that our proposed algorithm's complexity is much smaller.

4) We carry out a comprehensive performance analysis for the proposed algorithm. We show that, the gap between Pareto front generated by our algorithm and the real one is less than $0.16 \%$. This implies that, the proposed three-stage iterative algorithm can guarantee promising MEC offloading performances for the co-existence of CM-UEs and CP-UEs.

The remainder of this paper is organized as follows. In Section II, we introduce the system model, and provide the transmission model for mmWave MEC offloading. Then, we formulate the MOP in Section III and gives the problem analysis. Thereafter, we propose a low complexity three-stage iterative algorithm in Section IV. In addition, we introduce a typical multi-objective optimization algorithm NSGA-II in Section V, and compare the complexity of this algorithm with the proposed algorithm. Numerical results for evaluating the performance of the proposed schemes are provided in Section VI. The conclusions are made in Section VII.

\section{System ModeL}
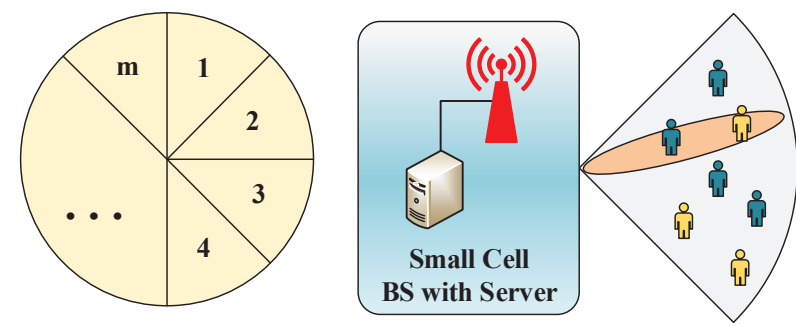

Sectors of

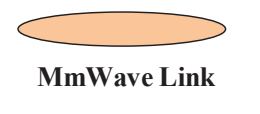

舟 CM-UE

MEC Small Cell

品 CP-UE

Fig. 1. Schematic for the mmWave MEC small cell.

Let us consider a mmWave MEC system in this paper. As shown in Fig. 1, we divide the MEC small cell into multiple sectors in order to achieve high utilization of spatial resources and to reduce the steering overhead of each mmWave beam [25-27]. We assume that adjacent sectors' transmissions operate on orthogonal frequency bands, so that inter-sector interference can be avoided. The BS is located in the center of the cell, and the UEs are randomly distributed in each sector, including $I \mathrm{CP}-\mathrm{UEs}$ and $J \mathrm{CM}$-UEs. The indexes of CP-UEs and CM-UEs are collected in sets $\mathcal{I}=\{1,2, \ldots, I\}$ and $\mathcal{J}=\{1,2, \ldots, J\}$ respectively. Our study focuses on the uplink transmission within one sector. Let us assume that, the offloading decisions have been made, where all the CPUEs involved need to offload their computing tasks to the MEC server. In particular, each CP-UE $i$ needs to complete the offloading task $C_{i}$ within its tolerable delay $D_{i}$, where $C_{i}$ is the data transmission task generated by the computing service of UE $i$. At the same time, for CM-UEs, they have the minimum transmission rate requirements.

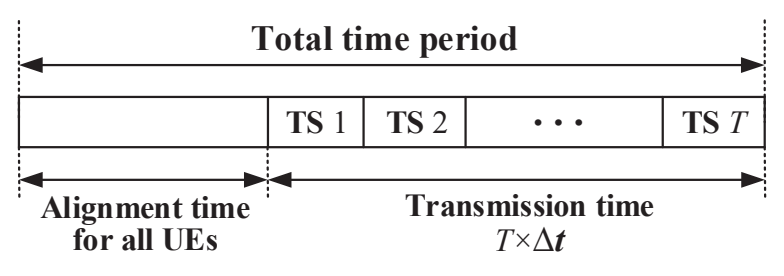

Fig. 2. The time period we considered.

As shown in Fig. 2, the total time period can be divided into two phases: beam allignment phase and data transmission phase. To meet multi-service requirement, we introduce the NOMA scheme for the data transmission phase. Without loss of generality, the transmission time is equally divided into $T$ TSs, and the indexes of TSs are included in set $\mathcal{T}=\{1,2, \ldots, T\}$, where the duration of each TS is $\Delta t$. The number of TSs satisfies $T=I=J$, and we assume that each CM-UE transmits in only one specific TS $t$. In each TS $t$, a CP-UE can be assigned to it and the active CP-UE 
and CM-UE in $t$ are regarded as a NOMA UE pair. In each $\mathrm{TS}$, each beam can only convey the transmission for a pair of NOMA UEs.

Based on the above assumptions and characteristics of mmWave [28], the effective antenna gain of uplink transmission can be expressed as

$$
G=g_{\mathrm{ue}} g_{\mathrm{bs}},
$$

where $g_{\mathrm{ue}}$ and $g_{\mathrm{bs}}$ are the main lobe gain for UE and BS respectively. Further, we have

$$
\begin{gathered}
g_{\mathrm{ue}}=\frac{2 \pi-\left(2 \pi-\theta_{\mathrm{ue}}\right) z_{\mathrm{ue}}}{\theta_{\mathrm{ue}}}, \\
g_{\mathrm{bs}}=\frac{2 \pi-\left(2 \pi-\theta_{\mathrm{bs}}\right) z_{\mathrm{bs}}}{\theta_{\mathrm{bs}}} .
\end{gathered}
$$

The side lobe gains $z_{\mathrm{ue}}$ and $z_{\mathrm{bs}}$ are fixed value which satisfy $0 \leq z_{\mathrm{ue}} \ll 1$ and $0 \leq z_{\mathrm{bs}} \ll 1$, respectively. Since active UEs are in different locations in each TS, if beam alignment is performed at BS side in each TS, a larger beam alignment overhead will be generated. Therefore, we assume that the beamwidth of BS $\theta_{\mathrm{bs}}$ is a fixed value that can cover the entire sector, and the beamwidth of UE $\theta_{\mathrm{ue}}$ is a variable to be optimized. As shown in Fig. 2, it should be noted that, the beam alignment of each UE is a time-consuming process, and this time can be denoted as

$$
d\left(\theta_{\mathrm{ue}}\right)=\left\lceil\frac{\Psi_{\mathrm{ue}}}{\theta_{\mathrm{ue}}}\right\rceil T_{p},
$$

where $T_{p}$ is the time required for a pilot transmission, $\Psi_{\text {ue }}$ is sector-level beamwidth at UE side.

Let us now consider the data transmission phase. Based on the above system model, for active UEs in TS $t$, we first decode the transmitted symbol of CP-UE, so that the interference of CM-UE link will not change in TS $t$. In addition, we assume that the decoding order based on the principle of successive interference cancellation (SIC) [29] does not change each UEs minimum signal-to-interferenceplus-noise ratio (SINR) requirement (i.e. minimum decoding threshold can be met), which is a commonly-used assumption in the existing works such as [30-32]. Therefore, the SINR for active UEs in TS $t$ can be given by:

$$
\begin{gathered}
\gamma_{i, t}=\frac{p_{i, t} G_{i, t}\left|h_{i, t}\right|^{2} L_{i}}{p_{j, t} G_{j, t}\left|h_{j, t}\right|^{2} L_{j}+N_{0}}, \\
\gamma_{j, t}=\frac{p_{j, t} G_{j, t}\left|h_{j, t}\right|^{2} L_{j}}{N_{0}},
\end{gathered}
$$

where $p_{i, t}$ and $p_{j, t}$ are transmission power for CP-UE $i$ and CM-UE $j$ in TS $t$. In the above equation, $L_{i}=d_{i}^{-\zeta}$ and $L_{j}=$ $d_{j}^{-\zeta}$ denotes the large-scale fading gain, where $d_{i}$ and $d_{j}$ is the distance between BS and UE $i$ and $j$ respectively, while $\zeta$ is the pathloss exponent. Note that, $h_{i, t}$ and $h_{j, t}$ are smallscale fading gains which follow independent Nakagami- $m$ fading. Furthermore, the background noise is characterized by a zeromean, complex Gaussian random variable with variance $N_{0}$.

\section{PROBLEM Formulation}

\section{A. Original Problem Formulation}

In this section, we formulate and analyze the MOP of resource allocation for mmWave MEC offloading.

Let us first define how to calculate the delay of CP-UEs. For each CP-UE $i$, we assume that it can complete the offloading task within the assigned TS $t$, and the delay of CP-UE $i$ can be expressed as

$$
\tau_{i}=\tau_{0}+\sum_{\forall t \in \mathcal{T}} a_{i, t}(t-1) \Delta t+\frac{C}{R_{i, t}},
$$

where $\tau_{0}$ is beam alignment time for all UEs. Since the beam alignment process of UEs is completed one by one, $\tau_{0}$ can be expressed as

$$
\begin{aligned}
\tau_{0} & =\sum_{\forall i \in \mathcal{I}}\left\lceil\frac{\Psi_{\mathrm{ue}}}{\theta_{i, t}}\right\rceil T_{p}+\sum_{\forall j \in \mathcal{J}}\left\lceil\frac{\Psi_{\mathrm{ue}}}{\theta_{j, t}}\right\rceil T_{p} \\
& \approx T_{p} \Psi_{\mathrm{ue}}\left(\sum_{\forall i \in \mathcal{I}} \frac{1}{\theta_{i, t}}+\sum_{\forall j \in \mathcal{J}} \frac{1}{\theta_{j, t}}\right),
\end{aligned}
$$

where $\theta_{i, t}$ and $\theta_{j, t}$ are beamwidth of CP-UE $i$ and CM-UE $j$. The indicator $a_{i, t}=1$ if CP-UE $i$ is active UE in TS $t$, otherwise, $a_{i, t}=0$. The second term of (7) is the waiting time of CP-UE $i$. Transmission rate $R_{i, t}$ in (7) and $R_{j, t}$ can be given below, where $B$ is the available bandwidth.

$$
\begin{aligned}
& R_{i, t}=B \log _{2}\left(1+\gamma_{i, t}\right), \\
& R_{j, t}=B \log _{2}\left(1+\gamma_{j, t}\right) .
\end{aligned}
$$

Then we consider the problem formulation. The objectives considered in this paper include: 1) minimizing the average delay of CP-UEs; 2) maximizing the total sum rate of CMUEs [33-35]. The problem can be expressed as:

$$
\begin{array}{r}
\mathbb{P} 0: \min _{\mathcal{A}, \mathcal{P}, \Theta}\left\{\frac{\sum_{i \in \mathcal{I}} \tau_{i}}{I}\right\}, \\
\max _{\mathcal{A}, \mathcal{P}, \Theta}\left\{\sum_{\forall j \in \mathcal{J}} R_{j, t}\right\} .
\end{array}
$$

subject to:

$$
\begin{aligned}
& a_{i, t}=\{0,1\}, \forall i \in \mathcal{I}, \forall t \in \mathcal{T}, \\
& \sum_{t \in \mathcal{T}} a_{i, t}=\sum_{i \in \mathcal{I}} a_{i, t}=1, \forall i \in \mathcal{I}, \forall t \in \mathcal{T}, \\
& \tau_{i} \leq D_{i}, \forall i \in \mathcal{I}, \\
& R_{j, t} \geq R_{0}, \forall j \in \mathcal{J}, \forall t \in \mathcal{T}, \\
& p_{i, t} \frac{C}{R_{i, t}} \leq \alpha_{i} E_{0}, \forall i \in \mathcal{I}, \forall t \in \mathcal{T}, \\
& p_{j, t} \Delta t \leq \beta_{j} E_{1}, \forall j \in \mathcal{J}, \forall t \in \mathcal{T}, \\
& \theta_{0} \leq \theta_{i, t} \leq \theta_{1}, \forall i \in \mathcal{I}, \forall t \in \mathcal{T}, \\
& \theta_{0} \leq \theta_{j, t} \leq \theta_{1}, \forall j \in \mathcal{J}, \forall t \in \mathcal{T} .
\end{aligned}
$$

Above, the objective functions are the average offloading delay of CP-UEs $\tau_{\mathrm{avg}}$ and the total transmission rate of CM-UEs $R_{\text {tot }}$, respectively. Note that, $\mathcal{A}=\left\{a_{i, t}, \forall i \in\right.$ $\mathcal{I}, \forall t \in \mathcal{T}\}$ contains the CP-UEs assignment variables, $\mathcal{P}=\left\{p_{i, t}\right.$ and $\left.p_{j, t}, \forall i \in \mathcal{I}, \forall j \in \mathcal{J}, \forall t \in \mathcal{T}\right\}$ contains power allocation variables and $\Theta=\left\{\theta_{i, t}\right.$ and $\theta_{j, t}, \forall i \in \mathcal{I}, \forall j \in$ $\mathcal{J}, \forall t \in \mathcal{T}\}$ contains beamwidth allocation variables.

As for constraints, (13) and (14) constrain that each CPUE needs to complete the offloading task within one TS, and each TS needs to be allocated one CP-UE. Further, (15) means each CP-UE $i$ should complete the offloading task within its tolerable delay $D_{i}$. In (16), the instantaneous rate of CM-UEs must be larger than $R_{0}$. We introduce $E_{0}$ in (17) and $E_{1}$ in (18) to limit the total energy consumption of CP-UEs and CM-UEs. In addition, $\alpha_{i}$ and $\beta_{j}$ are the energy consumption weight coefficients of CP-UE $i$ and CM-UE $j$, 
which satisfy $\sum_{i \in \mathcal{I}} \alpha_{i}=1$ and $\sum_{j \in \mathcal{J}} \beta_{j}=1$ respectively. Finally in (19) and (20), we give the beamwidth range of all UEs.

\section{B. Problem Analysis}

To find the Pareto optimal solutions of $\mathbb{P} 0$, we employ $\epsilon$-constrain method to transform this MOP into a SOP. By converting the second objective into a constraint with an adjustable upper bound, we can transform problem $\mathbb{P} 0$ into

$$
\mathbb{P} 1: \min _{\mathcal{A}, \mathcal{P}, \Theta}\left\{\tau_{\text {avg }}\right\},
$$

subject to:

$$
\begin{aligned}
& (13)-(20), \\
& -R_{\mathrm{tot}} \leq \epsilon=-R_{1},
\end{aligned}
$$

where $\tau_{\text {avg }}=\frac{\sum_{i \in \mathcal{I}} \tau_{i}}{I}$, and (21) means that total sum rate of CM-UEs should be greater than $R_{1}$.

Lemma 1: If $\tau_{\text {avg }}$ exists for all $(\mathcal{A}, \mathcal{P}, \Theta),\left(\mathcal{A}^{*}, \mathcal{P}^{*}, \Theta^{*}\right)$ is Pareto optimal for original MOP if and only if there exists $\epsilon$ such that $\left(\mathcal{A}^{*}, \mathcal{P}^{*}, \Theta^{*}\right)$ is the unique optimal solution to the SOP.

Proof: We first prove the sufficiency. Define that

$$
\begin{aligned}
& f_{1}(x)=\tau_{\mathrm{avg}}, \\
& f_{2}(x)=-R_{\mathrm{tot}},
\end{aligned}
$$

where $x=(\mathcal{A}, \mathcal{P}, \Theta)$. For a given $\epsilon$, assume $x^{*}$ is the unique optimal solution of the SOP, and we have $f_{1}\left(x^{*}\right) \leq f_{1}(x)$ for all $x$ satisfying the constraints (13) to (20). Then we assume that $x^{*}$ is not Pareto optimal for original MOP, which means there must exist another solution $x^{\prime}$ of problem SOP that satisfies

$$
f_{k}\left(x^{\prime}\right) \leq f_{k}\left(x^{*}\right), \quad \forall k=1,2,
$$

and there is at least one $l \in\{1,2\}$ such that $f_{l}\left(x^{\prime}\right)<f_{l}\left(x^{*}\right)$. Apparently, this contradicts with the uniqueness assumption. Therefore, we can conclude that $x^{*}$ is Pareto optimal for the original MOP. On the other hand, it is assumed that $x^{*}$ is a Pareto optimal solution for MOP. Then, let $\epsilon=f_{2}\left(x^{*}\right)$, and suppose that $x^{*}$ is not the optimal solution of SOP. Thus, there must exist another $x^{\prime}$, with $f_{1}\left(x^{\prime}\right) \leq f_{1}\left(x^{*}\right)$ and $f_{2}\left(x^{\prime}\right) \leq \epsilon=f_{2}\left(x^{*}\right)$, which contradicts with the assumption that $x^{*}$ is Pareto optimal for original MOP. The necessity is also proved.

From this lemma, it can be concluded that the $\epsilon$-constraint method is guaranteed to find all Pareto optimal solutions of the original MOP.

For convenience, we replace $1 / \theta_{i, t}$ and $1 / \theta_{j, t}$ with $\omega_{i, t}$ and $\omega_{j, t}$. According to (2), the antenna gain of UEs can be denoted as

$$
\begin{aligned}
G_{i, t} & =2 \pi\left(1-z_{\mathrm{ue}}\right) \omega_{i, t}+z_{\mathrm{ue}}, \\
G_{j, t} & =2 \pi\left(1-z_{\mathrm{ue}}\right) \omega_{j, t}+z_{\mathrm{ue}} .
\end{aligned}
$$

Considering the issue of fairness, we split the minimum sumrate $R_{1}$ in (21) according to the transmission rate requirements of each CM-UE. Combining (16), we can reformulate the problem as:

$$
\mathbb{P} 2: \min _{\mathcal{A}, \mathcal{P}, \Omega}\left\{\tau_{\text {avg }}\right\},
$$

subject to:

$$
\begin{aligned}
& a_{i, t}=\{0,1\}, \forall i \in \mathcal{I}, \forall t \in \mathcal{T}, \\
& \sum_{t \in \mathcal{T}} a_{i, t}=\sum_{i \in \mathcal{I}} a_{i, t}=1, \forall i \in \mathcal{I}, \forall t \in \mathcal{T}, \\
& \tau_{i} \leq D_{i}, \forall i \in \mathcal{I}, \\
& R_{j, t} \geq \max \left\{R_{0}, \gamma_{j} R_{1}\right\}, \forall j \in \mathcal{J}, \forall t \in \mathcal{T}, \\
& p_{i, t} \frac{C}{R_{i, t}} \leq \alpha_{i} E_{0}, \forall i \in \mathcal{I}, \forall t \in \mathcal{T}, \\
& p_{j, t} \Delta t \leq \beta_{j} E_{1}, \forall j \in \mathcal{J}, \forall t \in \mathcal{T}, \\
& \omega_{0} \leq \omega_{i, t} \leq \omega_{1}, \forall i \in \mathcal{I}, \forall t \in \mathcal{T}, \\
& \omega_{0} \leq \omega_{j, t} \leq \omega_{1}, \forall j \in \mathcal{J}, \forall t \in \mathcal{T} .
\end{aligned}
$$

where $\gamma_{j}$ in (31) represents the transmission rate weight coefficient of CM-UE $j$ and satisfies $\sum_{j \in \mathcal{J}} \gamma_{j}=1$. Further, $\omega_{0}$ and $\omega_{1}$ in (34) and (35) represent $1 / \theta_{0}$ and $1 / \theta_{1}$ respectively.

\section{Proposed Resource Allocation Scheme}

After converting the original MOP to $\mathbb{P} 2$, we can find that this problem is non-convex because of the integer variable $a_{i, t}$, transmission rate constraints and energy consumption constraints, which is NP-hard. Therefore, we propose to divide problem $\mathbb{P} 2$ into three subproblems, which are then solved by a novel three-stage iterative scheme.

\section{A. SCA-based Beamwidth Allocation}

In this subsection, we regard $\mathcal{A}$ and $\mathcal{P}$ as fixed values, and a SCA-based beamwidth allocation algorithm is proposed to obtain the beamwidth allocation result

First of all, we introduce an auxiliary variable $z$ to handle the objective function in $\mathbb{P} 2$, and reformulate $\mathbb{P} 2$ as:

$$
\mathbb{P} 3:\left\{\Omega^{*}\right\}=\arg \min _{\Omega, z}\left\{z \mid \mathcal{A}^{*}, \mathcal{P}^{*}\right\},
$$

subject to:

$$
\begin{aligned}
& \tau_{i} \leq D_{i}, \forall i \in \mathcal{I}, \\
& R_{j, t} \geq \max \left\{R_{0}, \gamma_{j} R_{1}\right\}, \forall j \in \mathcal{J}, \forall t \in \mathcal{T}, \\
& p_{i, t} \frac{C}{R_{i, t}} \leq \alpha_{i} E_{0}, \forall i \in \mathcal{I}, \forall t \in \mathcal{T}, \\
& p_{j, t} \Delta t \leq \beta_{j} E_{1}, \forall j \in \mathcal{J}, \forall t \in \mathcal{T}, \\
& \omega_{0} \leq \omega_{i, t} \leq \omega_{1}, \forall i \in \mathcal{I}, \forall t \in \mathcal{T}, \\
& \omega_{0} \leq \omega_{j, t} \leq \omega_{1}, \forall j \in \mathcal{J}, \forall t \in \mathcal{T}, \\
& \tau_{\text {avg }} \leq z
\end{aligned}
$$

Obviously, this problem is still non-convex due to the nonconvex constraints (36), (38) and (42). To tackle this, we introduce an auxiliary variable $x_{i}$, which satisfying

$$
x_{i} \leq \frac{p_{i, t} G_{i, t}\left|h_{i, t}\right|^{2} L_{i}}{p_{j, t} G_{j, t}\left|h_{j, t}\right|^{2} L_{j}+N_{0}} .
$$

In this case, (36), (38) and (42) can be re-written as

$$
\begin{gathered}
\tau_{0}+\tau_{0}^{\prime}+\frac{C}{B \log _{2}\left(1+x_{i}\right)} \leq D_{i}, \\
\frac{p_{i, t} C}{B \log _{2}\left(1+x_{i}\right)} \leq \alpha_{i} E_{0},
\end{gathered}
$$




$$
\tau_{0}+\frac{1}{I} \sum_{\forall i \in \mathcal{I}} \tau_{0}^{\prime}+\frac{C}{B I} \sum_{\forall i \in \mathcal{I}} \frac{1}{\log _{2}\left(1+x_{i}\right)} \leq z .
$$

Based on (7) and (8), $\tau_{0}$ and $\tau_{0}^{\prime}$ in (44) and (46) can be given by

$$
\begin{gathered}
\tau_{0}=\Psi_{\mathrm{ue}} T_{p}\left(\sum_{\forall i \in \mathcal{I}} \omega_{i, t}+\sum_{\forall j \in \mathcal{J}} \omega_{j, t}\right), \\
\tau_{0}^{\prime}=\sum_{\forall t \in \mathcal{T}} a_{i, t}(t-1) \Delta t .
\end{gathered}
$$

It can be easily verified that (44), (45) and (46) are now convex.

To handle the non-convex constraint (43), another auxiliary variable $y_{j}$ needs to be defined:

$$
x_{i} y_{j} \leq p_{i, t} G_{i, t}\left|h_{i, t}\right|^{2} L_{i},
$$

where

$$
y_{j} \geq p_{j, t} G_{j, t}\left|h_{j, t}\right|^{2} L_{j}+N_{0}
$$

The upper bound of $x_{i} y_{j}$ can be denoted as

$$
x_{i} y_{j} \leq \frac{y_{j}^{[n]}}{2 x_{i}^{[n]}} x_{i}^{2}+\frac{x_{i}^{[n]}}{2 y_{j}^{[n]}} y_{j}^{2},
$$

where $y_{j}^{[n]}$ and $x_{i}^{[n]}$ respectively denote the values of $y_{j}$ and $x_{i}$ at the $n$-th iteration. Then, (49) can be rewritten as the following convex constraint

$$
\frac{y_{j}^{[n]}}{2 x_{i}^{[n]}} x_{i}^{2}+\frac{x_{i}^{[n]}}{2 y_{j}^{[n]}} y_{j}^{2} \leq p_{i, t} G_{i, t}\left|h_{i, t}\right|^{2} L_{i} .
$$

Now we can reformulate problem $\mathbb{P} 3$ as:

$$
\mathbb{P} 4:\left\{\Omega^{*}\right\}=\arg \min _{\Omega, z, x_{i}, y_{j}}\left\{z \mid \mathcal{A}^{*}, \mathcal{P}^{*}\right\},
$$

subject to:

$$
(37),(40),(41),(44),(45),(46),(50),(52) \text {. }
$$

It is clear that $\mathbb{P} 4$ is a convex optimization problem, which can be solved by standard convex optimization technique, such as interior point method. Note that because of the existence of constraint (52), we need to iteratively solve $\mathbb{P} 4$ to obtain the solution of $\mathbb{P} 3$, and this will be explained in next subsection. It is worth noting that, when initializing $x_{i}$ and $y_{j}$, we assign the tolerable minimum value given by constraint (44) and (45) to $x_{i}^{[0]}$, and give any value of $y_{j}^{[0]}$ in the feasible region of $y_{j}$.

Remark 1: SCA-based algorithm can converge to the approximate optimal value.

Proof: We will prove the convergence through the change trend of the parameters $x_{i}^{[n]}$ and $y_{j}^{[n]}$ in each iteration.

For $y_{j}$, it is obvious that this variable does not directly affect the objective function $z$. However, in order to make the objective function $z$ smaller, $y_{j}$ will choose the smallest value in the feasible region at each iteration. Hence, it should satisfy

$$
y_{j}^{[0]} \geq y_{j}^{[1]}=y_{j}^{[q]}, q \geq 2 .
$$

By contrast, for $x_{i}$, in order to minimize $z$, in the first iteration, it needs to select the maximum value in the feasible region, giving that, $x_{i}^{[1]} \geq x_{i}^{[0]}$. The coefficient of $x_{i}^{2}$ becomes smaller, which means that $x_{i}$ can take a larger value to further reduce the objective function $z$. This is true for every iteration. Therefore, we can find that, the value of $z$ decreases or at least equal value as the number of iterations increases, and it clearly has a lower bound. Hence, the convergence of this SCA-based beamwidth allocation part can be proved. Similar work was also proved in [36].

\section{B. BCD-based Iterative Beamwidth and Power Allocation}

In this subsection, we first consider the power allocation problem while regarding $\mathcal{A}$ and $\Omega$ as fixed values. Problem $\mathbb{P} 2$ can be reformulated as

$$
\mathbb{P} 5:\left\{\mathcal{P}^{*}\right\}=\arg \min _{\mathcal{P}}\left\{\tau_{\text {avg }} \mid \mathcal{A}^{*}, \Omega^{*}\right\},
$$

subject to:

$$
\begin{aligned}
& \tau_{i} \leq D_{i}, \forall i \in \mathcal{I}, \\
& R_{j, t} \geq \max \left\{R_{0}, \gamma_{j} R_{1}\right\}, \forall j \in \mathcal{J}, \forall t \in \mathcal{T}, \\
& p_{i, t} \frac{C}{R_{i, t}} \leq \alpha_{i} E_{0}, \forall i \in \mathcal{I}, \forall t \in \mathcal{T}, \\
& p_{j, t} \Delta t \leq \beta_{j} E_{1}, \forall j \in \mathcal{J}, \forall t \in \mathcal{T},
\end{aligned}
$$

Based on (8) and (9), we can observe that the objective function $\tau_{\text {avg }}$ of $\mathbb{P} 5$ increases as $p_{j, t}$ gets bigger, or as $p_{i, t}$ gets smaller. At the same time, the lower bound of $p_{j, t}$ and upper bound of $p_{i, t}$ can be easily obtained from (55) and (56). The lower bound of $p_{j, t}$ can be expressed as:

$$
p_{j, t}^{\min }=\frac{\left(2^{\frac{R_{j}^{\max }}{B}}-1\right) N_{0}}{G_{j, t}\left|h_{j, t}\right|^{2} L_{j}},
$$

where $R_{j}^{\max }=\max \left\{R_{0}, \gamma_{j} R_{1}\right\}$. Upon applying $p_{i, t}$, we need to apply the result of (56)-(58), we derive

$$
\frac{p_{i, t} C}{B \log _{2}\left(1+\frac{p_{i, t} G_{i, t}\left|h_{i, t}\right|^{2} L_{i}}{p_{j, t}^{\min } G_{j, t}\left|h_{j, t}\right|^{2} L_{j}+N_{0}}\right)} \leq \alpha_{i} E_{0} .
$$

It is obviously that the left hand of (59) is a monotonically increasing function of $p_{i, t}$, the upper bound of $p_{i, t}$ can be readily found. Based on the above analysis, we can use Lambert-W function to find the optimal $p_{i, t}$. After some mathematical manipulations, we have $(60)$, where $W(x)$ is the Lambert-W function [36]. Then, it is easy to solve $\mathbb{P} 5$ and get the optimal solution $\mathcal{P}^{*}$.

To solve the problems of beamwidth allocation and power allocation, we propose an iterative algorithm for $\mathbb{P} 4$ and $\mathbb{P} 5$ by applying the block coordinate descent (BCD) method [37]. As suggested by [21] and [38], the BCD method can efficiently solve the multi-convex coupling problem, while guaranteeing low complexity with fast convergence. Specifically, the optimization variables are partitioned into two blocks, i.e., $\{\Omega, \mathcal{P}\}$. Then, these two blocks are alternately optimized by solving $\mathbb{P} 4$ and $\mathbb{P} 5$ correspondingly, while keeping the other block of variables fixed. Furthermore, the obtained solution in each iteration is used as the input of the next iteration. The details of this algorithm are summarized in Algorithm 1.

Lemma 2: BCD-based iterative beamwidth and power allocation algorithm can converge to an approximate optimal result.

Proof: It should be noted that in classical BCD method, the sub-problem for updating each block of variables is required to be solved exactly with optimality in each iteration in order to guarantee the convergence [37]. However, in our case, for the beamwidth optimization problem, we only 


$$
p_{i, t}^{\max }=-\frac{\alpha_{i} E_{0} B \cdot W\left(-\frac{C \ln 2\left(p_{j, t}^{\min } G_{j, t}\left|h_{j, t}\right|^{2} L_{j}+N_{0}\right)}{\alpha_{i} E_{0} B G_{i, t}\left|h_{i, t}\right|^{2} L_{i}} 2^{-\frac{C\left(p_{j, t}^{\min } G_{j, t}\left|h_{j, t}\right|^{2} L_{j}+N_{0}\right)}{\alpha_{i} E_{0} B G_{i, t}\left|h_{i, t}\right|^{2} L_{i}}}\right)}{C \ln 2}-\frac{p_{j, t}^{\min } G_{j, t}\left|h_{j, t}\right|^{2} L_{j}+N_{0}}{G_{i, t}\left|h_{i, t}\right|^{2} L_{i}}
$$

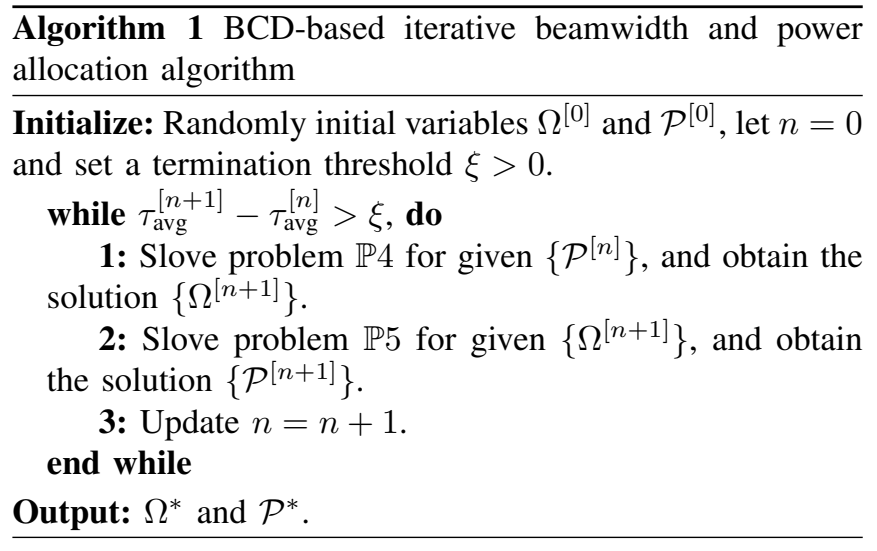

solve the approximate problems in $\mathbb{P} 4$. Thus, the convergence of the proposed BCD-based iterative beamwidth and power allocation algorithm is proved as follows.

Define $\eta\left(\Omega^{[n]}, \mathcal{P}^{[n]}\right)$ as the optimal objective value after the $n$-th iteration. First of all, in step 1 of Algorithm 1, since the optimal solution of $\mathbb{P} 4$ is obtained for given $\mathcal{P}^{[n]}$, we have

$$
\eta\left(\Omega^{[n]}, \mathcal{P}^{[n]}\right) \leq \eta\left(\Omega^{[n+1]}, \mathcal{P}^{[n]}\right) .
$$

Second, for given $\Omega^{[n+1]}$ in step 2 of Algorithm 1, the optimal solution of $\mathbb{P} 4$ is obtained. Then we have

$$
\eta\left(\Omega^{[n+1]}, \mathcal{P}^{[n]}\right) \leq \eta\left(\Omega^{[n+1]}, \mathcal{P}^{[n+1]}\right) .
$$

Based on (61)-(62), we obtain

$$
\eta\left(\Omega^{[n]}, \mathcal{P}^{[n]}\right) \leq \eta\left(\Omega^{[n+1]}, \mathcal{P}^{[n+1]}\right),
$$

which indicates that the objective value is non-decreasing after each iteration of Algorithm 1. Since the optimal value of $\tau_{\text {avg }}$ is lower bounded by a finite value, this algorithm can converge to a solution closing to the optimal one.

\section{O2OM CP-UE Assignment}

After introducing the issues of beamwidth allocation and power allocation, we can get two fixed variable sets, i.e., $\left\{\mathcal{P}^{*}, \Omega^{*}\right\}$. Upon applying the results, the problem of CP-UE assignment can be expressed as:

$$
\mathbb{P} 6:\left\{\mathcal{A}^{*}\right\}=\arg \min _{\mathcal{A}}\left\{\tau_{\text {avg }} \mid \mathcal{P}^{*}, \Omega^{*}\right\}
$$

subject to:

$$
\begin{aligned}
& a_{i, t}=\{0,1\}, \forall i \in \mathcal{I}, \forall t \in \mathcal{T}, \\
& \sum_{t \in \mathcal{T}} a_{i, t}=\sum_{i \in \mathcal{I}} a_{i, t}=1, \forall i \in \mathcal{I}, \forall t \in \mathcal{T},
\end{aligned}
$$

In order to find the optimal solution of $\mathbb{P} 6$, we propose the one-to-one matching (O2OM) algorithm. Let us introduce some important preliminaries below.

In the matching process, we define two disjoint finite sets: CP-UE set $\boldsymbol{I}$ and TS set $\boldsymbol{T}$, then a one-to-one matching $\phi$ is defined as

$$
\begin{aligned}
& \forall i \in \boldsymbol{I}, \phi(i) \in \boldsymbol{T}, \quad|\phi(i)|=1, \\
& \forall t \in \boldsymbol{T}, \phi(t) \in \boldsymbol{I}, \quad|\phi(t)|=1 .
\end{aligned}
$$

Combining constraints (64) and (65), $\phi(i)=t$ implies that $a_{i, t}=1$, otherwise $a_{i, t}=0$.

Based on problem $\mathbb{P} 6$, the utility functions for CP-UE $i$ and TS $t$ can be given by

$$
U_{i}=U_{t}=R_{i, t}
$$

where $R_{i, t}$ is the transmission rate of CP-UE $i$ in TS $t$. According to the definition of utility functions, the preference relationships $\succ_{t}$ and $\succ_{f}$ are defined as:

$$
\begin{aligned}
& i \succ_{t} i^{\prime} \Leftrightarrow U_{i}(\phi)>U_{i^{\prime}}\left(\phi^{\prime}\right), \\
& t \succ_{i} t^{\prime} \Leftrightarrow U_{t}(\phi)>U_{t^{\prime}}\left(\phi^{\prime}\right) .
\end{aligned}
$$

Futher, we introuduce the matching actions:

Swapping action: The CP-UE $i$ swaps the assigned time slot $t$ with another CP-UE $i^{\prime}$ assigned time slot $t^{\prime}$.

Based on the above preliminaries, the principles of $\mathrm{O} 2 \mathrm{OM}$ algorithm is summarized in Algorithm 2. We consider the process of solving problem $\mathbb{P} 6$ as the matching process of CP-UEs and TSs. Through iterative optimization of the matching relationship between two players, a stable matching is finally achieved, and the optimal UE allocation strategy is obtained. In addition, we incorporate the aforementioned BCD-based iterative beamwidth and power allocation algorithm into each swap iteration.

In a little more detail, Algorithm 2 consists three parts. In the first part, it randomly allocates the CP-UEs and obtains allocation matrix $\boldsymbol{A}$ and swap control matrix $\boldsymbol{C}$. Due to the fusion of Algorithm 1 with O2OM, we need to initialize variables $\Omega$ and $\mathcal{P}$. In the second part, we check the swap control list for each CP-UE and carry out swap actions. In the third part, Algorithm 1 is executed based on the given current allocation matrix $\boldsymbol{A}$.

Lemma 3: Algorithm 2 can achieve a stable matching result for $\mathrm{CP}-\mathrm{UEs}$ and TSs pairing.

Proof: The proof comes from three key facts. First of all, each CP-UE (or TS) can not be assigned to multiple TSs (or CP-UEs) during the considered time period. Therefore, the number of possible swaps is finite. Moreover, only the swaps which strictly increase the links utility can occur. Secondly, once all the possible swaps have been executed, this algorithm terminates and each CP-UE remains associated with the most preferred TS, and vice versa. Thirdly, Algorithm 1 can converge to a stable point at each iteration in matching process, and avarage delay for CP-UEs has a lower bound when we consider three variables: $\mathcal{A}, \mathcal{P}$, and $\Theta$. Hence the introduction of Algorithm 1 will not affect the convergence of the matching process. Therefore, Algorithm 2 can converge to a stable point. The proof of stable matching can also be found in [39] 


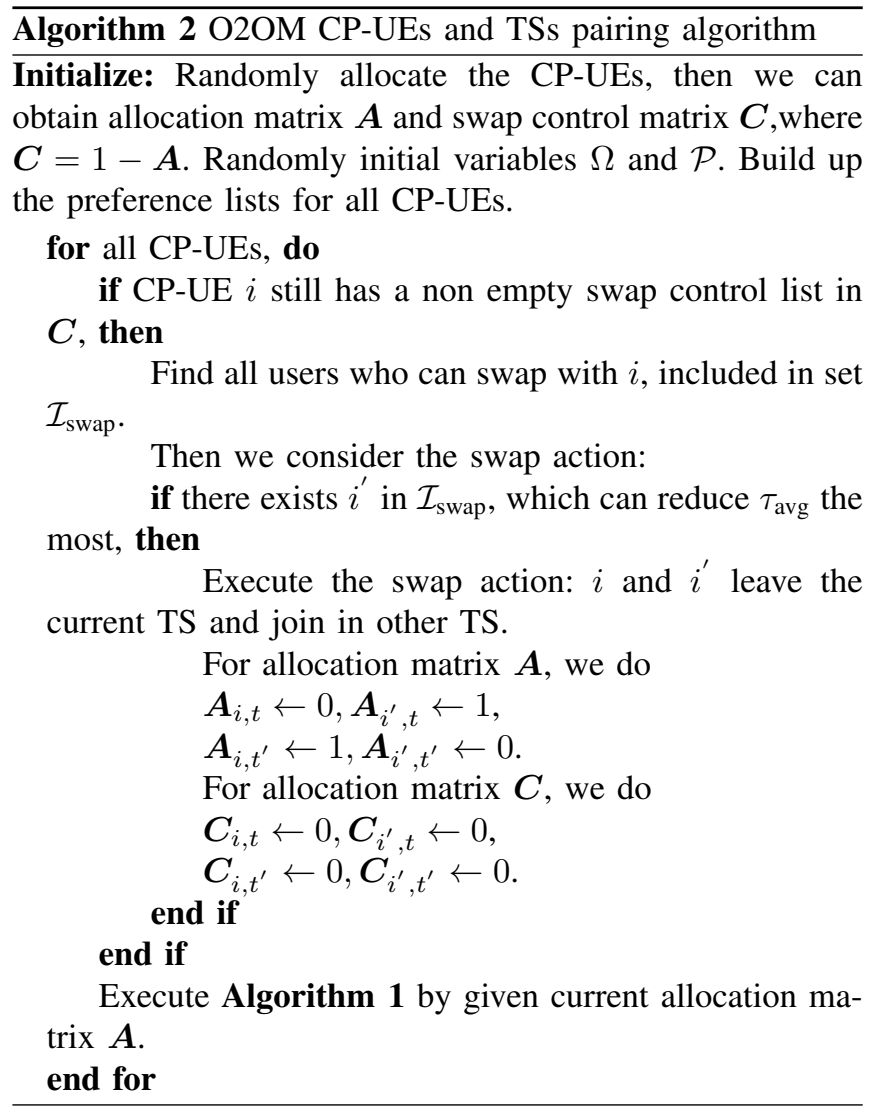

\section{BENCHMARK SCHEME AND COMPLEXITy ANALYSIS}

In this section, we introduce a typical multi-objective genetic algorithm, which is a benchmark scheme. Further, we provide the complexity analysis for our proposed schemes.

\section{A. Benchmark Scheme: NSGA-II}

Comparing with other intelligent algorithms, in this section, we introduce a typical multi-objective genetic algorithm: NSGA-II. Compared with other multi-objective optimization algorithms, this algorithm has a higher population diversity, and can obtain a more complete Pareto front. Moreover, NSGA-II is widely-accepted as a promising approach for solving the problem with low-dimensional multi-objectives and variables being strongly coupled.

However, the typical NSGA-II algorithm can not directly solve the MINLP. In order to apply the typicality of the NSGA-II algorithm, we transform the original MOP $\mathbb{P 0}$ into the following form:

$$
\begin{aligned}
\mathbb{P} 7: & \min _{\mathcal{A}, \mathcal{P}, \Theta}\left\{\tau_{\text {avg }}\right\}, \\
& \max _{\mathcal{A}, \mathcal{P}, \Theta}\left\{R_{\text {tot }}\right\} .
\end{aligned}
$$

subject to:

$$
\begin{aligned}
& 0 \leq a_{i, t} \leq 1, \forall i \in \mathcal{I}, \forall t \in \mathcal{T}, \\
& \sum_{t \in \mathcal{T}}\left\langle a_{i, t}\right\rangle=\sum_{i \in \mathcal{I}}\left\langle a_{i, t}\right\rangle=1, \forall i \in \mathcal{I}, \forall t \in \mathcal{T}, \\
& (15),(16),(17),(18),(19),(20) .
\end{aligned}
$$

In problem $\mathbb{P} 7$, we regard $\mathcal{A}$ as continuous variables, which can be constrained by (73) and (74). Simultaneously, $a_{i, t}$ that appears in (71) and (72) is represented by $\left\langle a_{i, t}\right\rangle$, where $\langle\cdot\rangle$ is the rounding function. This is for converting the discrete variables into continuous variables and adding nonlinear equality constraints to them.

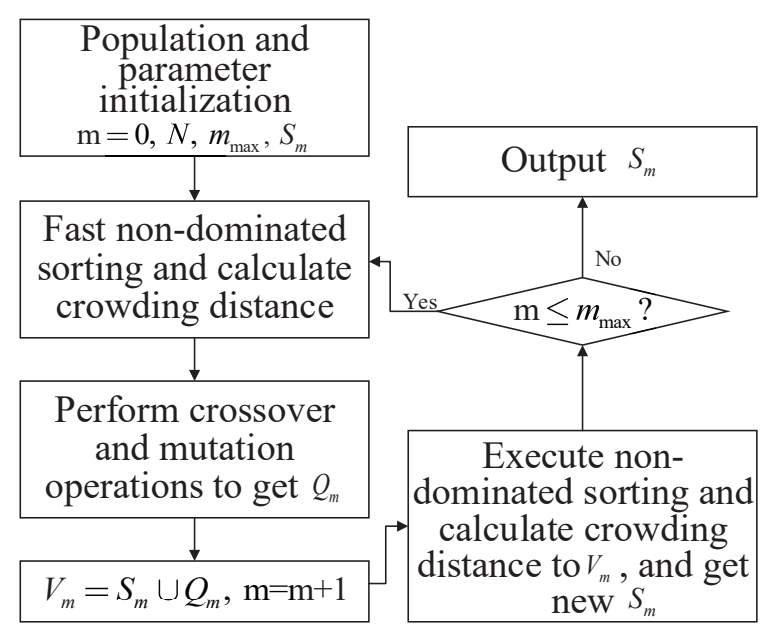

Fig. 3. The flowchart of NSGA-II algorithm

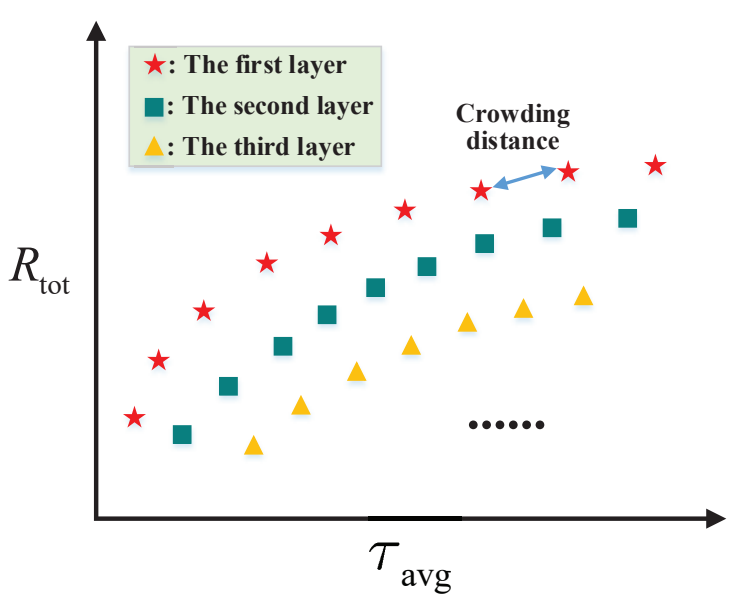

Fig. 4. Non-dominated set and crowding distance.

Then, we use typical NSGA-II algorithm to solve problem $\mathbb{P} 7$. As shown by Fig. 3, there are six main procedures. First of all, we randomly select $N$ groups of $\mathcal{A}, \mathcal{P}$ and $\Theta$, as well as obtaining the corresponding $\tau_{\text {avg }}$ and $R_{\text {tot }}$, i.e., the population $S_{m}$. At the same time, we initialize genetic counter $m=0$ and maximum genetic times $m_{\max }$. Then, the genetic operation on the population is executed, which is also the core of this algorithm. We perform the fast non-dominated sorting on each individual in $S_{m}$, and calculate the crowding distance in each layer. Specifically, as shown in Fig. 4, in the non-dominated set, the first layer is the set of non-dominated individuals of all, the second layer is the set of non-dominated individuals obtained after removing the individuals of the first layer, and so on. In other words, individuals on the upper level have better $\tau_{\text {avg }}$ and $R_{\text {tot }}$ performance than those on the lower level. As for the crowding distance, it is the distance between individuals in the same layer. Crossover and mutation operations are carried out on the selected upper layer individuals while the selection result is related to crowding distance so that we can obtain the new individuals set $Q_{m}$. Finally, it performs the non-dominated sorting and crowding distance calculating on the expanded population $V_{m}=S_{m} \cup Q_{m}$, then the new $S_{m}$ is obtained after elite-preserving selection based on the performance of $\tau_{\text {avg }}$ and $R_{\text {tot. }}$. After $m_{\max }$ iterations, it can derive the optimal Pareto front of two objectives in $\mathbb{P} 7$ and 
$\left\{\mathcal{A}^{*}, \mathcal{P}^{*}, \Theta^{*}\right\}$.

This algorithm has two typical characteristics. First of all, it define a partial order set according to the level and crowding-distance of individuals in current population, and select individuals in this set when constructing next generation. Second, the elite-preserving operator is applied to this algorithm, which is more conducive to retaining excellent individuals, thereby improving the overall evolutionary level of the population.

\section{B. Complexity Analysis}

In this subsection, we analyze the complexity of the proposed three-stage iterative algorithm and NSGA-II algorithm.

In our work, the time complexity of the algorithms, is reflected by the number of repeated executions of floating point operations [40]. Let us first discuss the three-stage iterative algorithm, which includes three parts: SCA-based beamwidth allocation, BCD-based iterative power allocation, and O2OM CP-UEs assignment. In beamwidth allocation part, we perform SCA to this sub-problem, turn it into a convex problem and solve it with the CVX toolbox. The core of the convex optimization toolbox is the interior point method, so that the complexity of this part in each BCD iteration is

$$
O\left([2(I+J)+1]^{3.5}\right)
$$

where $I$ and $J$ represent the number of CP-UEs and CM-UEs respectively. The power allocation part is directly solved by Lambert-W function in each BCD iteration. Therefore, the complexity of the entire BCD process (including beamwidth allocation and power allocation) can be expressed as

$$
O\left(M_{1}\left\{[2(I+J)+1]^{3.5}+1\right\}\right),
$$

where $M_{1}$ is the number of iterations of $\mathrm{BCD}$ process. Finally, the O2OM CP-UEs assignment is a solution based on matching algorithm. In the worst case, assuming that in each iteration, the optimal matching selected from the preference list always conflicts with other matching results, then each CP-UE matches at most $T$ times, and its complexity is $O(I T)$. In addition, the complexity of updating the preference list for each $\mathrm{CP}-\mathrm{UE}$ is $O(T \log T)$. Hence, the complexity of this part can be expressed as

$$
O\left(M_{2}(I T+I T \log T)\right),
$$

where $M_{2}$ is the number of iterations of $\mathrm{O} 2 \mathrm{OM}$ process, and $T$ is the number of TSs. Since the BCD process is nested in the matching process, the complexity of the entire algorithm satisfies the multiplication relationship. Therefore, the total complexity of the proposed three-stage iteration algorithm can be denoted as

$$
O\left(M_{1} M_{2}\left\{[2(I+J)+1]^{3.5}+1\right\}(I T+I T \log T)\right) .
$$

Based on section II, we have $I=J=T$, so the complexity of this algorithm can be approximately simplified to $O\left(T^{6}\right)$.

For the NSGA-II algorithm whose objective number is $r$ and population number is $N$, its complexity mainly comes from the following three parts [41]:

(1) Fast non-dominated sorting: $O\left(r(2 N)^{2}\right)$.

(2) Crowding distance assignment: $O(r(2 N) \log (2 N))$.

(3) Define partial order set: $O((2 N) \log (2 N))$.
Therefore, we can approximately simplify the complexity of this algorithm to $O\left(m_{\max } r N^{2}\right)$, where $m_{\max }$ is the maximum genetic operations.

It is worth noting that, there is no clear calculation method for the population size $N$ and maximum genetic times $m_{\max }$ of genetic algorithms. In order to make an intuitive complexity comparison, we give TABLE I based on the empirical value during simulation.

TABLE I

THE SMALLEST $N$ AND $m_{\text {MAX }}$ THAT CAN GET REAL PARETO FRONT

\begin{tabular}{ccc}
\hline \hline No. of TS $T$ & Population size $N$ & Maximum genetic times $m_{\max }$ \\
\hline 2 & 400 & 100 \\
4 & 1800 & 800 \\
6 & 3900 & 1900 \\
8 & 9000 & 3500 \\
10 & 19000 & 5700 \\
\hline
\end{tabular}

According to the data comparison in TABLE I, we can easily find that the complexity of the proposed three-stage iterative algorithm is much lower than NSGA-II algorithm.

\section{Simulation Result}

In this section, we provide the simulation results for the three-stage iterative algorithm in NOMA assisted mmWave MEC system, and compare with other algorithms. The key system parameters are shown in TABLE II.

TABLE II

SYSTEM PARAMETERS

\begin{tabular}{lll}
\hline \hline Notation & Parameter & Value \\
\hline$I$ & No. of CP-UEs & 8 \\
$J$ & No. of CM-UEs & 8 \\
$T$ & No. of TSs & 8 \\
$B$ & Available bandwidth & $2 \mathrm{GHz}$ \\
$\Psi_{\mathrm{ue}}$ & Sector-level beamwidth & $\pi / 2 \mathrm{rad}$ \\
$\Delta t$ & Duration per TS & $1 \mathrm{~s}$ \\
$T_{p}$ & Pilot transmission time & $1 \mathrm{~ms}$ \\
$\theta_{\mathrm{bs}}$ & Beamwidth of BS & $\pi / 4 \mathrm{rad}$ \\
$z_{\mathrm{bs}}$ & Side lobe gain of BS & 0.001 \\
$z_{\mathrm{ue}}$ & Side lobe gain of UE & 0.001 \\
\hline \hline
\end{tabular}

First of all, we compare the Pareto fronts obtained by NSGA-II algorithm with that obtained by the three-stage iterative algorithm in Fig. 5, when considering different energy consumption constraints. NSGA-II algorithm can approach the optimal Pareto boundary infinitely as the increase of iterations [41]. In the case of the same energy consumption constraints, the performance of the three-stage iterative algorithm is slightly worse than the NSGA-II algorithm, but the gap between these two Pareto fronts less than $0.16 \%$. This gap is caused by the sub-optimality of the SCA and BCD algorithm, which are proved in Remark 1 and Lemma 2. Furthermore, with the reduction of energy consumption constraints, the gaps between two kinds of Pareto fronts are getting smaller and smaller. With the reduction of the remaining energy at the UE side, the feasible range of variables shrinks, and traditional intelligent algorithms cannot accurately search for the best feasible solution. The core of the three-stage iterative algorithm is a value-based optimization process, which is less affected by the feasible 


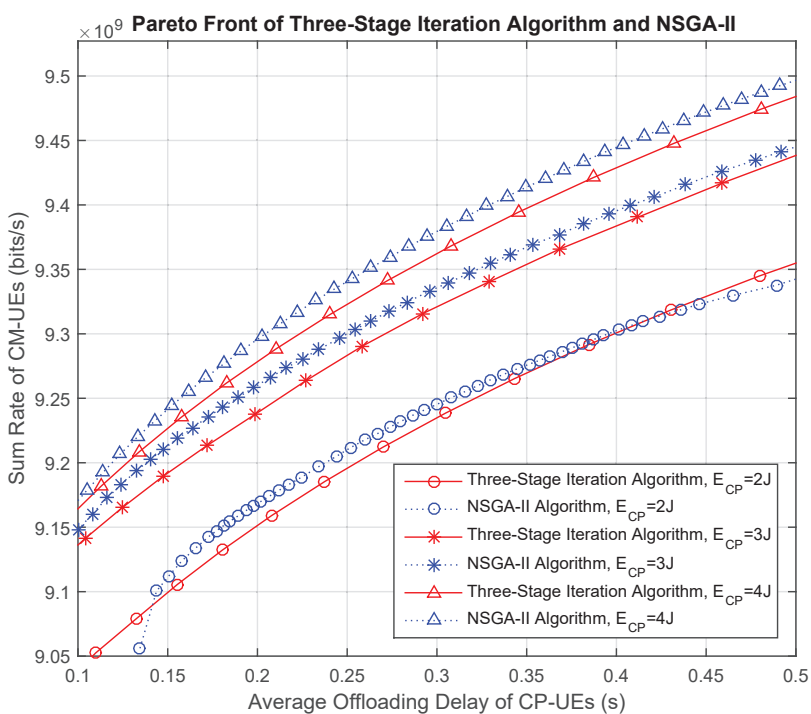

Fig. 5. Comparison of intelligent algorithm and three-stage iterative algorithm algorithm.

range, so that we can conclude that the proposed algorithm can achieve performance closer to the real Pareto front under the condition of limited energy consumption than intelligent algorithms. In addition, the proposed algorithm is better than NSGA-II in terms of the complexity of obtaining the Pareto front at the boundary. For our proposed algorithm, The multi-objective problem is solved by $\epsilon$-constraint method, which is operated in three-stage iteratively regardless of the boundary or the center points. Therefore, the complexity of the proposed algorithm in solving any point in the Pareto front is the same. By contrast, for the NSGA-II algorithm, it needs to increase the population size to a very large number, so that the diversity requirement for approaching Pareto front could be met. This will cause the exponential increase of complexity for the NSGA-II algorithm at the boundary points. In summary, the three-stage iterative algorithm is better than traditional intelligent algorithms in terms of time complexity and boundary performance.

Optimal Beamwidth Allocation under Different Energy Constraints

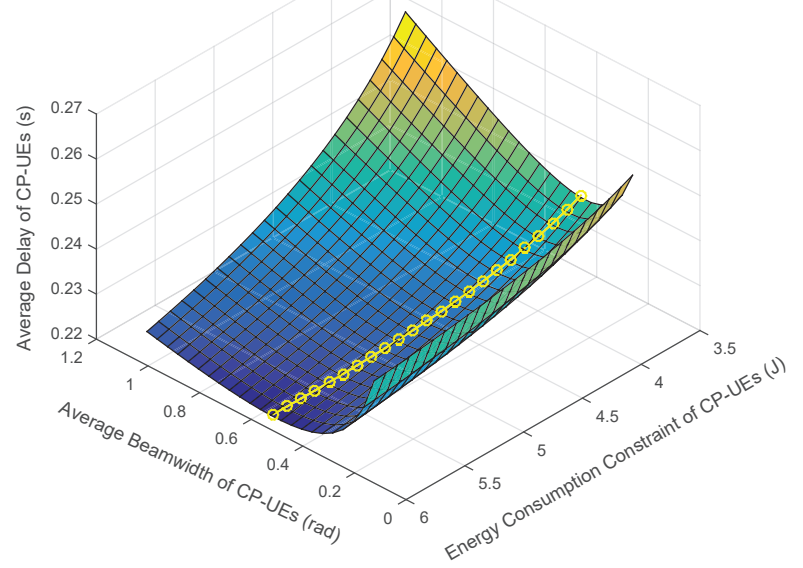

Fig. 6. The effect of beamwidth on system performance.

In this work, the beamwidth of mmWave link is regarded as a new dimension of resource allocation. In Fig. 6, we obtained a three-dimensional diagram to illustrate the rela- tionship between beamwidth and objective function under different energy consumption constraints. The best tradeoff result is marked as the circle point in this figure. It can be observed that, under certain energy consumption constraints, the average delay of CP-UEs first decreases and then increases as the beamwidth decreases. This is because although a narrower beam can achieve a higher transmission rate, it will also bring a longer beam alignment time. Hence, there is a trade-off relationship between transmission delay and beam alignment delay, which is also the significance of studying beamwidth allocation. The above findings imply that, in practical systems, the beamwidth of mmWave MEC link needs to be carefully optimized to balance the beam alignment delay and antenna gain desired.

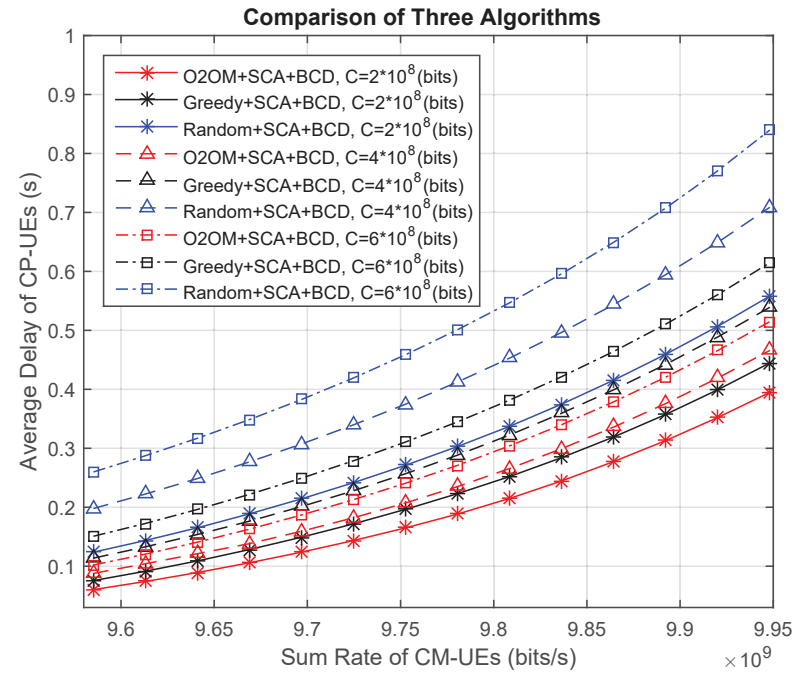

Fig. 7. The advantages of $\mathrm{O} 2 \mathrm{OM}$ algorithm.

In Fig. 7, we compare three kinds of CP-UEs and TSs pairing algorithms under different offloading tasks for $\mathrm{CP}$ UEs. First of all, we can find that as the transmission rate of CM-UEs increases, the average delay of CP-UEs gradually increases, which is caused by the competition for limited communication resources between CM-UEs and CP-UEs. Secondly, under a certain amount of offloading task, we can obverse that $\mathrm{O} 2 \mathrm{OM}$ algorithm can always achieve a lower average delay of CP-UEs than greedy and random pairing algorithms, regardless of the transmission rate of CMUEs. In addition, the gaps between $\mathrm{O} 2 \mathrm{OM}$ and greedy (or random) algorithm become larger as the sum rate increases, which shows that our proposed O2OM algorithm can achieve better performance in both objectives. Thirdly, as the value of the sum rate of CM-UEs increases, the average delay gaps between these three kinds of algorithms become bigger. This is because the traditional greedy and random allocation strategies can not meet the reasonable resource allocation when the two objectives exist at the same time, whereas the proposed O2OM algorithm can achieve better performances of CM-UEs and CP-UEs simultaneously.

In Fig. 8, we simulate and show the relationship between average offloading tasks and average offloading delay of CP-UEs under different energy consumption $E_{C P}$. It is obviously seen that, with the increase of offloading tasks, the average offloading delay increases exponentially, which is caused by the competition between CM-UEs and CPUEs for communication resources. More importantly, the gap 


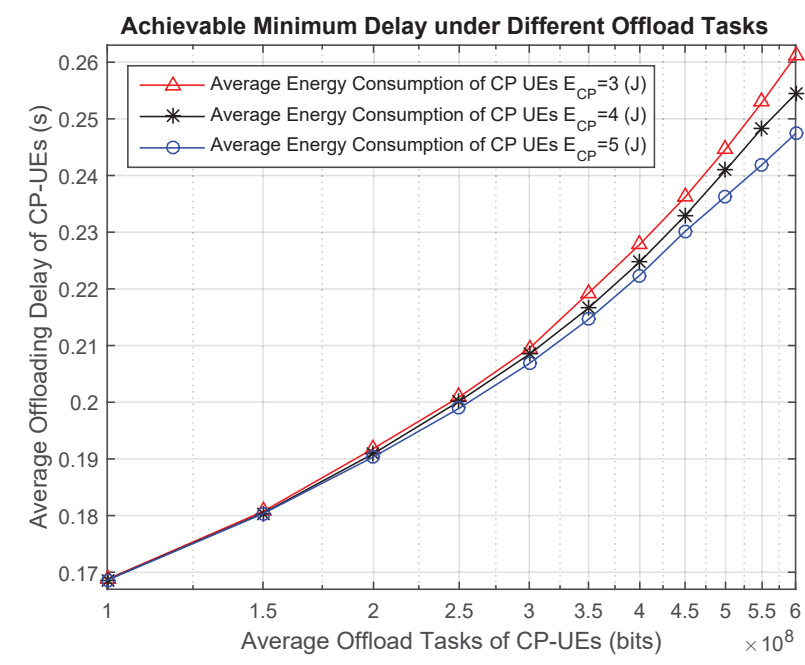

Fig. 8. The impact of offloading tasks on performance.

between different energy consumption constraints of CP-UEs increases rapidly with the increase of offloading tasks. This is because that, when the energy consumption is reduced, CP-UEs have to allocate a narrower beamwidth to meet the transmission delay requirement, which will bring a larger beam alignment delay. This means that when the available energy of CP-UEs decreases, the average offloading delay will increase substantially as the offloading tasks increases, which will cause CP-UEs can not complete the offloading tasks within delay tolerance. Therefore, practical resource management algorithms are particularly important to mobile edge UEs, and our proposed algorithm can successfully make the offloading delay within one TS.

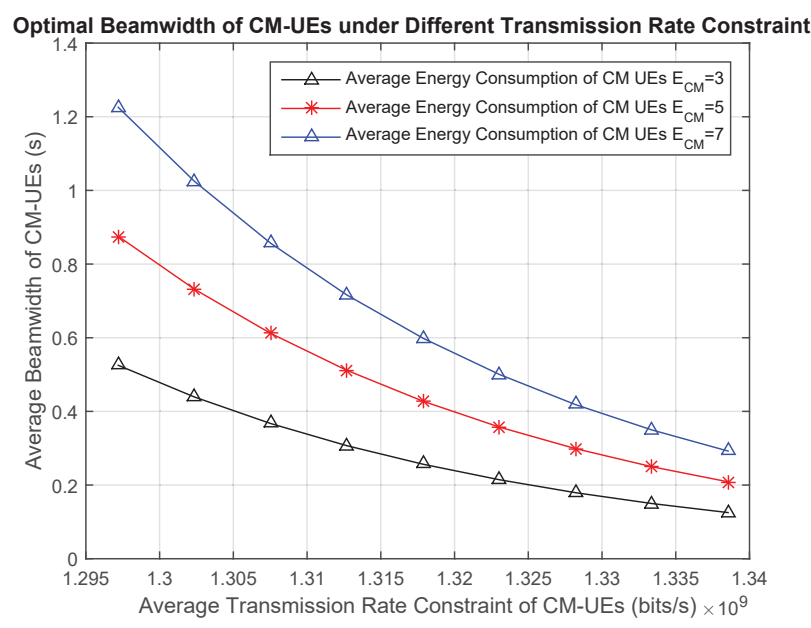

Fig. 9. Performance of CM-UEs under the three-stage iterative algorithm.

In Fig. 9, we show the beamwidth allocation results of the proposed algorithm under different average transmission rate constraints. We can observe that the average beamwidth of CM-UEs decreases as average transmission rate constraint increases, this is because in section III, we transform the second objective function in $\mathbb{P} 0$ into the constraint in $\mathbb{P} 1$ by $\epsilon$-constrain method. In order to obtain the minimum beam alignment delay, three-stage iterative algorithm will assign the maximum beamwidth under the conditions of the minimum CM-UEs transmission rate. Under certain transmission rate constraints, when the available energy consumption of CM-UEs increases, the transmission power increases when the transmission time is fixed, which will increase the beamwidth allocated to CM-UEs by the proposed algorithm, thereby reducing the beam alignment delay. In addition, When the average transmission rate of CM-UEs is less constrained, users can increase their own transmission power to achieve the lowest transmission rate. This will result in a larger difference in beamwidth under different energy consumption constraints. However, when the average transmission rate constraint of CM-UEs become larger, it is impossible to meet the minimum transmission rate requirement only by adjusting the power constraint. This will result in a rapid narrowing of the beamwidth, which in turn reduces the beamwidth gap under different energy consumption constraints. Hence, it can conclude that, the proposed three-stage iterative algorithm fully considers the offloading delay while meeting the transmission rate constraints of CM-UEs.

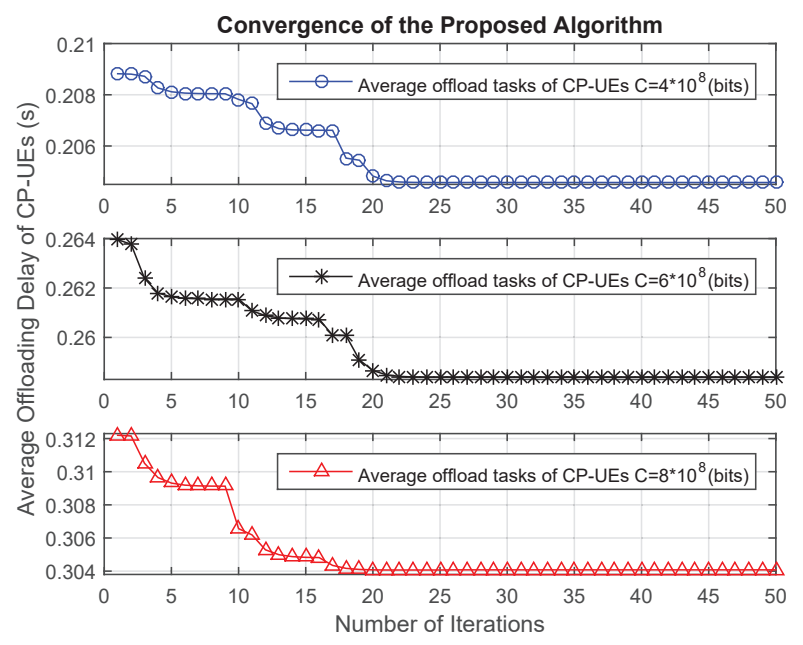

Fig. 10. The convergence process of the three-stage iterative algorithm.

Finally, in Fig. 10, we give the convergence of the threestage iterative algorithm. In order to express the convergence process of the entire algorithm more clearly, we choose the central iteration of the overall algorithm, SCA algorithm as the node, and calculate the average offloading delay after SCA is called. Obviously, after the SCA algorithm is awakened about 20 iterations, the entire algorithm can converge to a stable state. The sudden drop of average delay in the previous iterations is the performance mutation caused by the BCD algorithm iterating under the new pairing conditions after the O2OM algorithm is executed. Compared with NSGA-II, our proposed algorithm can achieve rapid convergence under different offloading tasks.

\section{CONCLUSION}

In this paper, we have investigated the resource management for the mmWave MEC offloading system, when considering the data transmission demands from both CMUEs and CP-UEs. The MOP of resource allocation has been formulated, and has been converted into a SOP. Based on that, we have proposed the three-stage iterative algorithm, which includes BCD-based iterative beamwidth and power allocation and O2OM CP-UE assignment. Our simulation results have shown that, the gap between Pareto front generated 
by three-stage iterative resource allocation algorithm and the real Pareto front less than $0.16 \%$. In addition, the proposed algorithm with much lower complexity can significantly outperforms the other traditional schemes. Therefore, due to the proposed algorithm exploiting the feature of lowcomplexity, it has great advantages in future mmWave MEC large-scale access scenarios, such as bustling streets, large shopping malls, stadiums, etc., where high-volume of computing services are demanded. In conclusion, the proposed three-stage iterative algorithm may constitute a promising candidate for resource allocation in practical MEC offloading with mmWave communication capability.

\section{REFERENCES}

[1] J. Cao, W. Feng, N. Ge and J. Lu, "Delay Characterization of MobileEdge Computing for 6G Time-Sensitive Services," in IEEE Internet of Things Journal, vol. 8, no. 5, pp. 3758-3773, 1 March1, 2021.

[2] N. Abbas, Y. Zhang, A. Taherkordi and T. Skeie, "Mobile Edge Computing: A Survey," in IEEE Internet of Things Journal, vol. 5, no. 1, pp. 450-465, Feb. 2018.

[3] F. Fang and X. Wu, "A WinCWin Mode: The Complementary and Coexistence of 5G Networks and Edge Computing," in IEEE Internet of Things Journal, vol. 8, no. 6, pp. 3983-4003, 15 March15, 2021.

[4] H. Li, H. Xu, C. Zhou, X. L and Z. Han, "Joint Optimization Strategy of Computation Offloading and Resource Allocation in Multi-Access Edge Computing Environment," in IEEE Transactions on Vehicular Technology, vol. 69, no. 9, pp. 10214-10226, Sept. 2020.

[5] Z. Ding, J. Xu, O. A. Dobre and H. V. Poor, "Joint Power and Time Allocation for NOMA MEC Offloading," in IEEE Transactions on Vehicular Technology, vol. 68, no. 6, pp. 6207-6211, June 2019.

[6] L. P. Qian, B. Shi, Y. Wu, B. Sun and D. H. K. Tsang, "NOMA-Enabled Mobile Edge Computing for Internet of Things via Joint Communication and Computation Resource Allocations," in IEEE Internet of Things Journal, vol. 7, no. 1, pp. 718-733, Jan. 2020.

[7] J. Zhao, Q. Li, Y. Gong and K. Zhang, "Computation Offloading and Resource Allocation For Cloud Assisted Mobile Edge Computing in Vehicular Networks," in IEEE Transactions on Vehicular Technology, vol. 68, no. 8, pp. 7944-7956, Aug. 2019.

[8] Y. Du, K. Yang, K. Wang, G. Zhang, Y. Zhao and D. Chen, "Joint Resources and Workflow Scheduling in UAV-Enabled Wirelessly-Powered MEC for IoT Systems," in IEEE Transactions on Vehicular Technology, vol. 68, no. 10, pp. 10187-10200, Oct. 2019.

[9] J. Feng, Q. Pei, F. R. Yu, X. Chu, J. Du and L. Zhu, "Dynamic Network Slicing and Resource Allocation in Mobile Edge Computing Systems,' in IEEE Transactions on Vehicular Technology, vol. 69, no. 7, pp. 7863 7878, July 2020.

[10] S. Yu, R. Langar, X. Fu, L. Wang and Z. Han, "Computation Offloading With Data Caching Enhancement for Mobile Edge Computing," in IEEE Transactions on Vehicular Technology, vol. 67, no. 11, pp. 11098-11112, Nov. 2018.

[11] S. Yu, B. Dab, Z. Movahedi, R. Langar and L. Wang, "A SociallyAware Hybrid Computation Offloading Framework for Multi-Access Edge Computing," in IEEE Transactions on Mobile Computing, vol 19, no. 6, pp. 1247-1259, 1 June 2020.

[12] M. Xiao et al., "Millimeter Wave Communications for Future Mobile Networks," in IEEE Journal on Selected Areas in Communications, vol. 35, no. 9, pp. 1909-1935, Sept. 2017.

[13] P. Zhou et al., "IEEE 802.11ay-Based mmWave WLANs: Design Challenges and Solutions," in IEEE Communications Surveys \& Tutorials, vol. 20 , no. 3, pp. 1654-1681, thirdquarter 2018.

[14] S. A. Busari, K. M. S. Huq, S. Mumtaz, L. Dai and J. Rodriguez, "Millimeter-Wave Massive MIMO Communication for Future Wireless Systems: A Survey," in IEEE Communications Surveys \& Tutorials, vol. 20, no. 2, pp. 836-869, Secondquarter 2018

[15] Z. Zhao, J. Shi, Z. Li, L. Yang, Y. Zhao and W. Liang, "Matching Theory Assisted Resource Allocation in Millimeter Wave Ultra Dense Small Cell Networks," in 2019 IEEE International Conference on Communications (ICC), Shanghai, China, 2019, pp. 1-6.

[16] Y. Liu, J. Liu, A. Argyriou and S. Ci, "MEC-Assisted Panoramic VR Video Streaming Over Millimeter Wave Mobile Networks," in IEEE Transactions on Multimedia, vol. 21, no. 5, pp. 1302-1316, May 2019.

[17] G. K. Tran et al., "Architecture of mmWave Edge Cloud in 5GMiEdge," in 2018 IEEE International Conference on Communications Workshops (ICC Workshops), Kansas City, MO, 2018, pp. 1-6.

[18] Q. Hu, C. Wu, X. Zhao, X. Chen, Y. Ji and T. Yoshinaga, "Vehicular Multi-Access Edge Computing With Licensed Sub-6 GHz, IEEE 802.11p and mmWave," in IEEE Access, vol. 6, pp. 1995-2004, 2018.
[19] S. Barbarossa, E. Ceci, M. Merluzzi and E. Calvanese-Strinati, "Enabling effective Mobile Edge Computing using millimeterwave links," in 2017 IEEE International Conference on Communications Workshops (ICC Workshops), Paris, 2017, pp. 367-372.

[20] S. Barbarossa, E. Ceci and M. Merluzzi, "Overbooking radio and computation resources in mmW-mobile edge computing to reduce vulnerability to channel intermittency," in 2017 European Conference on Networks and Communications (EuCNC), Oulu, 2017, pp. 1-5.

[21] C. Zhao, Y. Cai, A. Liu, M. Zhao and L. Hanzo, "Mobile Edge Computing Meets mmWave Communications: Joint Beamforming and Resource Allocation for System Delay Minimization," in IEEE Transactions on Wireless Communications, vol. 19, no. 4, pp. 2382-2396, April 2020.

[22] Y. Yu, X. Bu, K. Yang, H. Yang and Z. Han, "UAV-Aided Low Latency Mobile Edge Computing with mmWave Backhaul," in 2019 IEEE International Conference on Communications (ICC), Shanghai, China, 2019, pp. 1-7.

[23] Y. Chen, B. Ai, Y. Niu, Z. Zhong and Z. Han, "Energy Efficient Resource Allocation and Computation Offloading in Millimeter-Wave based Fog Radio Access Networks," in 2020 IEEE International Conference on Communications (ICC), Dublin, Ireland, 2020, pp. 1-6.

[24] N. Nouri, J. Abouei, M. Jaseemuddin and A. Anpalagan, "Joint Access and Resource Allocation in Ultradense mmWave NOMA Networks With Mobile Edge Computing," in IEEE Internet of Things Journal, vol. 7, no. 2, pp. 1531-1547, Feb. 2020.

[25] S. Sun, G. R. MacCartney, M. K. Samimi, S. Nie and T. S. Rappaport, "Millimeter wave multi-beam antenna combining for 5G cellular link improvement in New York City," in 2014 IEEE International Conference on Communications (ICC), 2014, pp. 5468-5473.

[26] Z. Wei, L. Zhao, J. Guo, D. W. K. Ng and J. Yuan, "Multi-Beam NOMA for Hybrid mmWave Systems," in IEEE Transactions on Communications, vol. 67, no. 2, pp. 1705-1719, Feb. 2019.

[27] Y. Liu, X. Fang, M. Xiao and S. Mumtaz, "Decentralized Beam Pair Selection in Multi-Beam Millimeter-Wave Networks," in IEEE Transactions on Communications, vol. 66, no. 6, pp. 2722-2737, June 2018.

[28] J. Wildman, P. H. J. Nardelli, M. Latva-aho and S. Weber, "On the Joint Impact of Beamwidth and Orientation Error on Throughput in Directional Wireless Poisson Networks," in IEEE Transactions on Wireless Communications, vol. 13, no. 12, pp. 7072-7085, Dec. 2014.

[29] D. Wang, N. Zhang, Z. Li, F. Gao and X. Shen, "Leveraging High Order Cumulants for Spectrum Sensing and Power Recognition in Cognitive Radio Networks," in IEEE Transactions on Wireless Communications, vol. 17, no. 2, pp. 1298-1310, Feb. 2018.

[30] J. Shi, W. Yu, Q. Ni, W. Liang, Z. Li and P. Xiao, "Energy Efficient Resource Allocation in Hybrid Non-Orthogonal Multiple Access Systems," in IEEE Transactions on Communications, vol. 67, no. 5, pp. 3496-3511, May 2019.

[31] Y. Zhang, H. Wang, T. Zheng and Q. Yang, "Energy-Efficient Transmission Design in Non-orthogonal Multiple Access," in IEEE Transactions on Vehicular Technology, vol. 66, no. 3, pp. 2852-2857, March 2017.

[32] Z. Yang, Z. Ding, P. Fan and N. Al-Dhahir, "A General Power Allocation Scheme to Guarantee Quality of Service in Downlink and Uplink NOMA Systems," in IEEE Transactions on Wireless Communications, vol. 15, no. 11, pp. 7244-7257, Nov. 2016.

[33] B. Bertenyi, 3GPP Release 15, Apr. 2019, [online] Available:https://www.3gpp.org/release-15

[34] P. Mogensen, A. Ghosh, A. Maeder, M. Uusitalo and S Redana, "5G evolution: A view on cellular technology beyond 5G", 6G Conf, Mar. 2019, Sep. 2019, [online] Available: http://www.6gsummit.com/speakers/preben-mogensen/.

[35] B. Bertenyi, 5G Standardization Update. 3GPP Webinar, Jul. 2019, [online] Available: https://www.3gpp.org/news-events/2058-ran-rel-16progress-and-rel-17-potential-work-areas.

[36] M. Zeng, W. Hao, O. A. Dobre and H. V. Poor, "Delay Minimization for Massive MIMO Assisted Mobile Edge Computing," in IEEE Transactions on Vehicular Technology, vol. 69, no. 6, pp. 6788-6792, June 2020.

[37] Xu Y, Yin W, "A block coordinate descent method for regularized multiconvex optimization with applications to nonnegative tensor factorization and completion," in SIAM Journal on imaging sciences, vol 6, no. 3, pp. 1758-1789, Sep. 2013.

[38] Q. Wu, Y. Zeng and R. Zhang, "Joint Trajectory and Communication Design for Multi-UAV Enabled Wireless Networks," in IEEE Transactions on Wireless Communications, vol. 17, no. 3, pp. 2109-2121, March 2018.

[39] Bodine-Baron, E., Lee, C., Chong, A., Hassibi, B., Wierman, A. "Peer effects and stability in matching markets", in International Symposium on Algorithmic Game Theory pp. 117-129, Oct. 2011.

[40] O. Goldreich, "Computational complexity: a conceptual perspective," Cambridge University Press, 2008, pp. 58-77. 
[41] Kalyanmoy Deb, "An efficient constraint handling method for genetic algorithms," in Computer Methods in Applied Mechanics and Engineering, vol 186, no. 2-4, pp. 311-338, Jun 2000. 